\title{
Multi-Agent System Architecture for Heart Failure Management in a Home Care Environment
}

\author{
VG Koutkias, I Chouvarda, N Maglaveras \\ Aristotle University, The Medical School, Lab. of Medical Informatics, Thessaloniki, Greece
}

\begin{abstract}
Health status management of heart failure patients is a rather complex and difficult procedure. Currently, a wellpromising approach facilitating this aim is home care systems, which provide monitoring, educational and surveillance services over several communication platforms, guaranteeing continuity and quality of care. In this paper, a Multi-Agent System architecture is proposed to support heart failure management in a generic home care setting. The system incorporates various processing modules based on statistics, rules and models that are applied to the medical data of patients, as well as learning capabilities based on the medical interventions that take place, and negotiation schemes among agents. The ultimate goal of this system is the characterization of the patients' health status and accordingly the notification of the corresponding medical personnel to take medical actions.
\end{abstract}

\section{Introduction}

Heart failure (HF) is a clinical syndrome, associated with very high rates of morbidity and mortality, which has become more prevalent in recent years. The costs related to $\mathrm{HF}$ management, encompassing hospitalization and outpatients' visits, are difficult to assess. Behavioral factors, such as noncompliance with diet and medications, social factors, such as social isolation, and lack of education about the disease, frequently contribute to early readmissions. These readmissions are a particularly appropriate target for intervention, because the population at risk is identified and the factors that contribute to this phenomenon are well known [1]. Published reports suggest that multidisciplinary programs have the potential to help prevent these early readmissions [2].

The aim of this study is to develop an agent-based system that assists the intensified treatment, monitoring and guidance of patients with HF through the use of a home care system and towards reduced HF-related hospitalization and mortality. The use of medical contact centers that offer home care services and act as mediators between medical staff and citizens seeking advice and/or therapy is a popular approach to modern IT-based healthcare delivery. The main objective of these home care systems is to provide monitoring, surveillance and educational services to patients suffering from chronic diseases through several communication platforms [3].

In such a home care scenario for HF management, the medical personnel have to monitor the patient's status and accordingly regulate him/her with clinical actions. Therefore, new requirements are emerging towards supporting the home care services and especially the medical personnel of the contact centers, due to the increased workload and complexity introduced by the large volume of multi-modal data collected, during the patient sessions with the contact center. Specifically, efficient information extraction and monitoring mechanisms are required for the effective management and automatic interpretation of monitored data. On the other hand, whenever needed, the discovered knowledge must be delivered to the healthcare personnel on time.

In this paper, a Multi-Agent System (MAS) architecture is proposed [4], aiming to constitute an advanced mechanism for the management of HF-patients that participate in the services offered by a home care telemedicine system and specifically by a contact center. The MAS continuously monitors medical data submitted during the patient sessions with the contact center, identifies patterns of abnormality almost instantly and, when necessary, notifies the medical personnel accordingly by generating reports, alerts, recommendations, etc. More precisely, the monitoring procedure consists of a multi-stage processing mechanism applied to medical data, which takes into account the medical interventions (e.g. drug change, hospital visits, etc.) registered in the contact center, as well as the medical history of the patients, in order to generate a more accurate characterization of the patient's condition for the medical personnel. To implement this functional scenario, software agents were adopted, due to their autonomous, reactive and/or proactive nature, their ability to apply negotiation strategies, when a decision has to be made, their communicative skills among them, and the learning mechanisms that may be embodied into them [4]. These features are considered favorable for deploying applications in dynamic, complex and distributed environments like the one described. 
An interesting agent-based approach for automated patient monitoring in intensive care units has been presented in [5]. However, the adoption of such a system in a home care setting, and telemedicine applications in general, is considered innovative and interesting. MASs constitute a novel approach towards collaborative problem solving in dynamic and complex environments, such as a home care system for HF-patients, where the process of decision making depends on a variety of parameters, which are often contradictory and have to be effectively assessed.

\section{Methods}

\subsection{Heart failure home care system}

Communication between patients and the HF contact center is preferably provided by a Computer Telephony application, although other communication means may be also available, such as Wireless Technology, or Internet Technology. The description of a common patient interaction with the HF contact center follows. As soon as the patient connects to the service, he/she may choose to initiate a session, i.e. submit his/her daily measurements, browse educational content or leave a message for the corresponding cardiologist. Cardiologists involved with the contact center, define the schedule and the content of the various session types for each patient, offering services customized to medical profiles.

During the measurements session, each patient may send the measurements of his/her vital parameters, e.g. blood pressure, temperature, etc., or bio-signals such as ECG, which are taken at home using simple microdevices (e.g. a 1-lead ECG recorder trans-telephonic transmission device) [6]. Complementary to the measurements can be a number of questions asked to the patients, related with current lifestyle and possible symptoms -signs, since the corresponding answers may be explanatory of their current health condition. As far as education is concerned, educational content is typically structured in categories related with, e.g. "Exercise", "Therapeutic Treatment", etc. Patients can access educational messages (e.g. in hypertext or voice format) among several categories, customized by their cardiologists, according to their specific medical profile. Another part of the educational content consists of tips, i.e. small messages that advise patients on certain issues. These pieces of prompting/advice can be delivered to the patient during a session or asynchronously. Furthermore, depending on the communication media, text or voice messages can be exchanged between the patient and his/her cardiologist.

Cardiologists review patient data on a regular basis and decide for new interventions, i.e. medication modifications, patient education and prompting, hospital visit or hospitalization. Frequent monitoring and intervention can improve patient regulation and decrease hospitalization rates. Independently of the communication means used, all the data flowing to/from the contact center are stored in specialized Computerized Patient Records, specifically designed to meet the functional requirements of the home care system.

\subsection{Multi-agent system functionality and design}

In order to implement an efficient mechanism for interpreting the monitored parameters of each patient and for notifying the cardiologists promptly in cases where abnormal conditions occur, a MAS architecture is proposed, in accordance with the HF home care scenario described above. This approach constitutes an automated way of coping with the increased workload and complexity introduced by the large volume of multimodal data collected, during the patient sessions with the contact center. Our agent-based framework is consisted of various purpose software agents, which embody the features depicted in Fig.1. The basic tasks applied by agents are:

- Medical data access, for monitoring the patient status in a real-time mode

- Medical data processing, for patient data interpretation

- Reasoning, in order to evaluate the outcome of the processing procedure

- Communication with other agents of the framework, for agent-to-agent collaboration and knowledge sharing

- Negotiation for decision making, in order to assess the individual outcome of each specific agent and generate an overall characterization of the patient status, and

- Learning capabilities, for improving the reasoning capabilities of agents and the system's performance

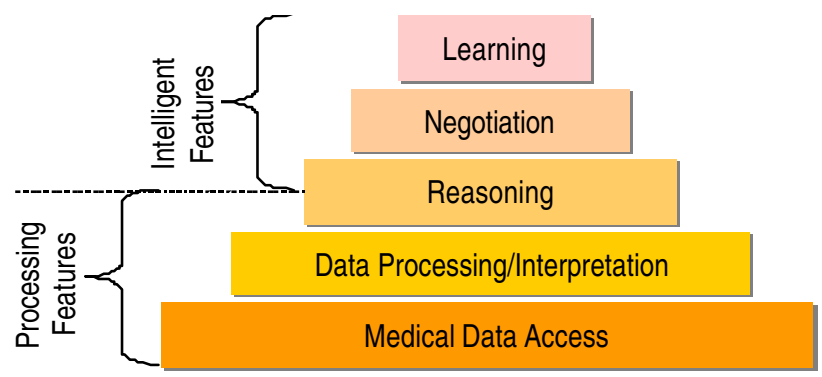

Figure 1. A hierarchical representation of the agent tasks.

The proposed MAS has been designed by delegating each monitoring parameter to a specific agent type, providing this way a specialization to each agent's role. Decomposition of tasks into simple ones was a 
fundamental design principle, in order to decrease the computational burden assigned to each agent and specialize its capabilities [7]. In addition, coordination among agents of the MAS is achieved through the exchange of appropriate messages.

According to the medical parameters that they have to monitor, during the patient sessions established with the contact center, the following agent-types may be distinguished:

- Agents monitoring a discrete value parameter, e.g. systolic blood pressure

- Agents monitoring a continuous-time parameter, such as ECG signals, and

- Agents monitoring a lifestyle-related parameter, such as the answer to the Yes/No question: "Did you take your medication?"

Appropriate processing techniques are applied, based on the medical parameters monitored. More specifically, medical rules and statistics are applied for the interpretation of discrete value and lifestyle-related parameters (based on thresholds, expected answers and consecutive sessions with the contact center). Signal processing techniques are adopted for the analysis of continuous-time parameters (e.g. ECG), in order to identify possible abnormalities. Moreover, the agents take into account the medical history of the patients, as well as the interventions applied by the corresponding cardiologists, in the processing procedure. Thus, a more advanced outcome towards the characterization of patient status may be accomplished.

Each agent of the MAS that applies a processing mechanism incorporates a different reasoning scheme, constituting a knowledge base, to monitor the different type of information independently. Therefore, different symptoms may be identified and their combination generates the overall result. Thus, a negotiation protocol among the agents of the framework is incorporated, in order to assess the results generated by each agent's activity alone, and conclude with an overall outcome. A negotiation plan is required, due to the contradictory results that may be generated and the need to generate a more accurate outcome. Negotiation is based on the significance of the parameters for each patient, which is defined by the cardiologists, and on the overall outcome, which has to be medically sound. This way the entire processing mechanism applied in the MAS constitutes a multi-stage processing procedure.

A learning model is also proposed as part of the framework, aiming to interrelate the interventions applied by the cardiologists with the agent-based outcome, in order to enhance the efficacy of the automated monitoring system, adapting the artificial model to human actions.

It has to be noted, that since the agent activity has to be applied within several terminals of the contact center (e.g. cardiologist's terminal, contact center medical database, etc.), agents of the MAS are distributed in the framework of the healthcare organization's Intranet providing the home care services. For security reasons, access to the medical data of the home care system is provided through a specialized wrapper agent (mediation service) that authenticates other agents of the framework and serves their requests. In Fig. 2, the MAS architecture is depicted, emphasizing on the system's functionality.

\section{Results}

In the current paper, a MAS architecture is proposed for $\mathrm{HF}$ management in home care systems. Most of the features described above have been developed in the context of the CHS home care system [6], such as the medical data access mechanism, the processing mechanisms incorporated to each different agent type, as well as their reasoning schemes. As an agent platform, JADE (Java Agent DEvelopment Framework) was adopted, which is compliant with the interoperability specifications of FIPA (Foundation of Intelligent Physical Agents) and provides both an agent execution environment and the means to interconnect different distributed platforms [8]. JADE platforms are installed wherever agents have to operate. The reasoning schemes of the agents are constructed by u sing JESS, a rule engine and scripting environment implemented in Java that enables the embodiment of "reasoning" in Java-based applications by using knowledge supplied in the form of declarative rules [9]. Messages among the agents of the framework are expressed in the FIPA ACL agent communication language.

Currently, spectrumbased Heart Rate Variability (HRV) is adopted for ECG processing/interpretation, indicated as HF/LF, which shows the balance between high and low frequencies of the heart rate power spectrum. Decrease of this parameter indicates deterioration in patient's condition. HF patients show depressed HRV, due to derangement in cardiac autonomic control. In general, higher HRV is desirable; lower HRV has been found to be a significant predictor of cardiac mortality and morbidity [10].

As far as the agent negotiation protocol is concerned, it is currently being under development. The design of a model, such as a cost-function, in which all the corresponding parameters will be taken into account in order to generate the overall processing outcome, is investigated. Such a negotiation mechanism has to be extensively evaluated with various test cases.

The learning feature described has to be developed and incorporated in the agents of the MAS. Techniques such as statistical or reinforcement learning are seriously considered for adoption [11].

MASs are difficult to evaluate, because there are no specific metrics, widely accepted, for the assessment of their architectural integrity and the performance of their implementation. In our case, the most important key- 
point would be the MAS's contribution to the medical procedure. The proposed system certainly undertakes a workload, and processes it almost instantly, while humans usually perform such tasks periodically and need a significant amount of time and effort to accomplish, thus, losing sometimes the best opportunities for intervention. A more elaborate evaluation plan would also include the survey of the users' (cardiologists, patients) overall satisfaction, by use of a questionnaire, or a more extended record of the cardiologist's reaction towards every individual instance of intervention.

\section{Conclusion}

Heart failure is a chronic disease that requires continuous monitoring and regulation. Home care monitoring is a favorable solution, however, adding workload and information complexity to the cardiologists. The proposed system introduces an innovation to such services, adding the notions of automated monitoring in a proactive way, which leads to an initial patient triage system. It is a step towards the design of intelligent, flexible and integrated home care telemedicine systems, easing the increased work of medical personnel, optimizing the time needed for data interpretation and enabling cardiologists to focus their attention on the management of abnormal cases.

\section{Acknowledgements}

This work was supported in part by the IST-199913352 Project, entitled 'Distance Information Technologies for Home Care - The Citizen Health System (CHS)' and the IST-2001-33369 Project, entitled 'Citizen Centered Health and Lifestyle Management via Interactive TV: The PANACEIA Health System', both funded by the CEC.

\section{References}

[1] Gooding J, Jette AM. Hospital readmissions among the elderly. J Am Geriatr Soc 1985;33:595-601.

[2] Rich MW, Beckham V, Wittenberg C, Leven CL, Freedland KE, Carney RM. A multidisciplinary intervention to prevent the readmission of elderly patients with congestive heart failure. $\mathrm{N}$ Engl $\mathrm{J}$ Med 1995;333:1190-5.

[3] Horowitz JD. Home-based intervention: the next step in treatment of chronic heart failure? Eur Heart J 2000;21:1807-9.

[4] Sycara K. Multiagent Systems. AI Magazine 1998;10 (2):79-93.

[5] Katehakis D, Chalkiadakis G, Tsiknakis M, Orphanoudakis S. A distributed, agent-based architecture for acquisition, management, archiving and display of realtime monitoring data in the Intensive Care Unit. FORTHICS Technical Report TR-261, Heraklion, Greece, 1999.

[6] Maglaveras $\mathrm{N}$ et al. Using Contact Centers in TeleManagement and Home Care of Congestive Heart Failure Patients - The CHS Experience, Computers in Cardiology 2002;29:281- 4

[7] Koutkias VG, Chouvarda I, Maglaveras N. Agent-based Monitoring and Alert Generation for a Home Care Telemedicine System. In: Proc. of AMIA Annual Symposium 2002. San Antonio, Texas, 2002;395-9.

[8] Bellifimine F, Poggi A, Rimassa G. JADE - A FIPAcompliant agent framework. In: Proc. of the 4th International Conference and Exhibition on the Practical Application of Intelligent Agents and Multi-Agents 1999. 1999:97-108.

[9] Java Expert System Shell (JESS). Available at: http://herzberg.ca.sandia.gov/jess/

[10] Bonaduce D, Petretta M, Marciano F, Vicario ML, Apicella C, Rao MA, Nicolai E, Volpe M. Independent and Incremental Prognostic Value of Heart Rate Variability in Patients with Chronic Heart Failure. Am Heart J 1999 Aug;138(2 Pt 1):273-84.

[11] Rusell SJ, Norvig P. Artificial Intelligence: A Modern Approach $2^{\text {nd }}$ Ed. 2003. New Jersey: Prentice Hall.

Address for correspondence.

Nicos Maglaveras, $\mathrm{PhD}$

Associate Professor

Aristotle University - The Medical School

Lab. of Medical Informatics - Box 323

54124 Thessaloniki, GREECE

EMAIL : nicmag@med.auth.gr

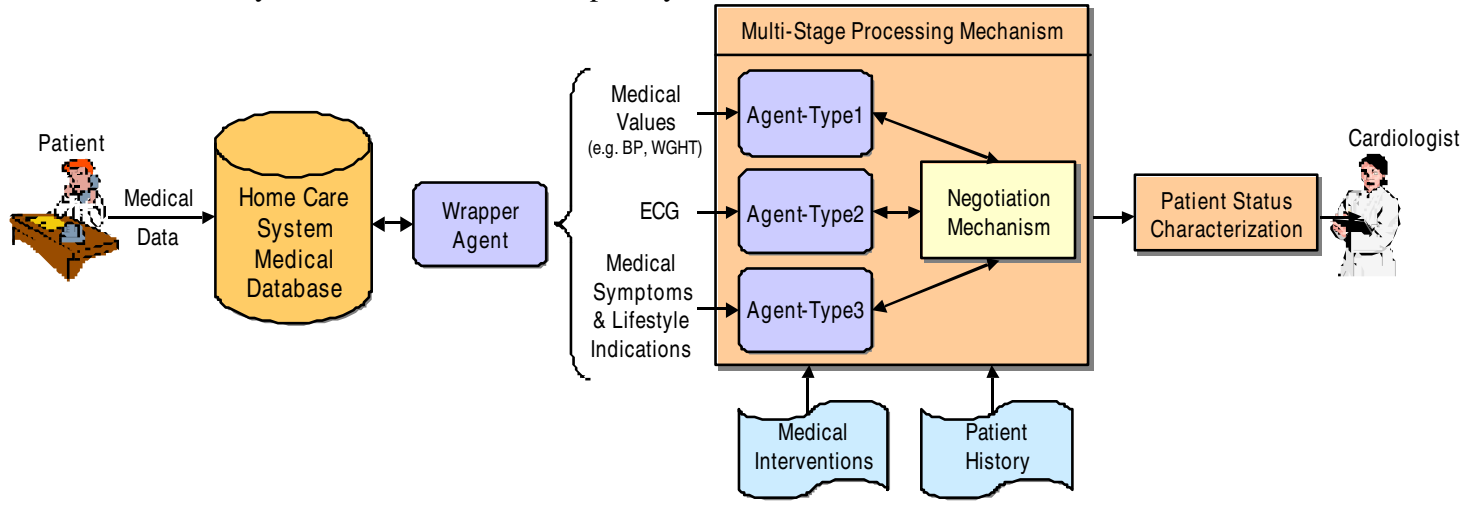

Figure 2. The architecture of the proposed MAS for HF management in a home care system. 